\title{
Imaios.com: “e-MRI” Reloaded and More
}

\section{Ozdoba}

Published online: 9 January 2013

(C) Springer-Verlag Berlin Heidelberg 2012

Three and a half years ago, in issue 3/2009 of "Clinical Neuroradiology," I presented and recommended the website "e-mri.org" in this column. Today, this site is no longer accessible.

The contents, however, are not lost to the web community.

\section{Imaios.com: Anatomy and MRI}

The contents of the former site www.e-mri.org are now found at www.imaios.com. However, they are a kind of addendum to the site's primary focus which is on anatomy.

Some content is available for free, but the majority requires a subscription (cf. Fig. 1). The different subscription options are explained in detail on the website.

As our residents love Imaios (Fig. 2 shows why), we have an institutional subscription that grants access to users that come to the site from a defined IP address.
The free content - some of the anatomic information and the complete magnetic resonance imaging (MRI) course that was formerly found at www.e-mri.org - is fully accessible only with a registration (Fig. 3). This registration is free; the operators of this site just want to know who visits them. In my opinion, the MRI course alone is worth the little trouble to login with a username and password whenever you access the site.

In summary, Imaios is well worth the money. When dictating a report, the detailed anatomy studies often come in handy especially (but not limited to) for residents in training, and the quality of the MRI course has remained unchanged.

C. Ozdoba $(\bowtie)$

Bern, Switzerland

e-mail: christoph.ozdoba@insel.ch 


\section{Head and Neck Anatomy}

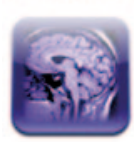

Brain (MRI 3D)

Anatomy of the encephalon in MRI (axial, coronal and sagittal slices)

Anatomical structurest cerebral lobes, fegions, telencephalon (loses, association fibres of the white martor, central grey nucleil, diencephalon, cerebellum, brainstem, nuclei of cranial nerves, ventricles, meningess, vascular 353 MR images | 26 anatomy diagrams | 524 anatomical parts

\section{Brain (MRI in axial slices) FREE}

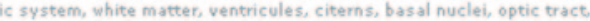
cerebellum, brainstem, arteries, venous sinuses, glands, spinal cord.

144 MR images 160 anatomical parts

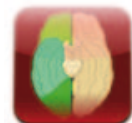

Brain (diagrams)

Anotazed illustrations of the anatomy of the bran

matomical structures: lobes and cerebral tegrons, telencephalon, diencephalon, cerebellum, clusters of whit: matter, ventricles, arteries, veins, meninges, cranial nerves, functional areas (Brodmann):

64 anatomy diagrams 730 anatomical parts

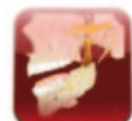

Cranial nerves (diagrams)

\section{Daranium}

Emergence and foramen of the cranial nerves, trajectories of the nerves, organs and related structures.

15 anatomy diagrams | 280 anatomical parts

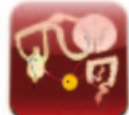

\section{Autonomic nervous system (diagrams) FREE}

cross-section of a ganglion and spinal nerves, sympathetic and parasympathetic innervation of organs, autonomic system of the face and cranial nerves

3 anatomy diagrams | 150 anatomical parts

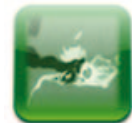

Petrous bone (CT)

Aranemes reructurest external ear, ossicles, tympanic cavity, anterior and posterior bony labyrinth, temporal ils crimages

$115 \mathrm{CT}$ images 110 anatomical parts

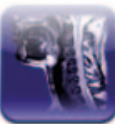

Face and neck (MRI)

Anatomical structures: bones, force and neck based on MRT in axial, coronal and sagittal slices.

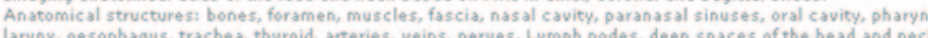
418 MR images | 505 anatomical parts

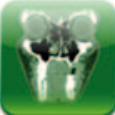

\section{Face (CT) Garaniont}

Anatomical imagery atlas of the face and base of the skull in scan.

Anatomical structures: benes, teeth, arteries, veins, muscles, nerves, foramen, cavities, mucous membranes Alands, ganglia

$420 \mathrm{CT}$ images | 310 anatomical parts

Fig. 1 Imaios offers a wide choice of anatomic images. Besides computed tomography $(\mathrm{CT})$ and magnetic resonance (MR) studies, instructive diagrams are available for special regions. The selections marked
e-Anatomy quicklinks
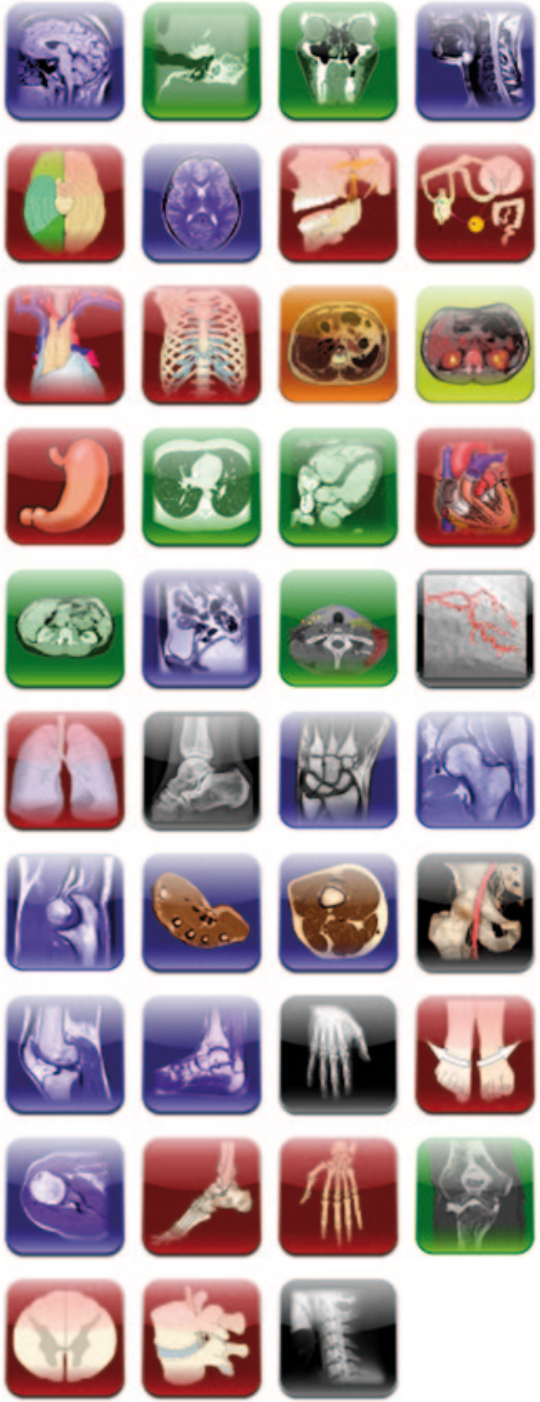

as "free" are free for registered users, the "premium" topics require a chargeable subscription (C) IMAIOS SAS (last time accessed on 6th December 2012) 

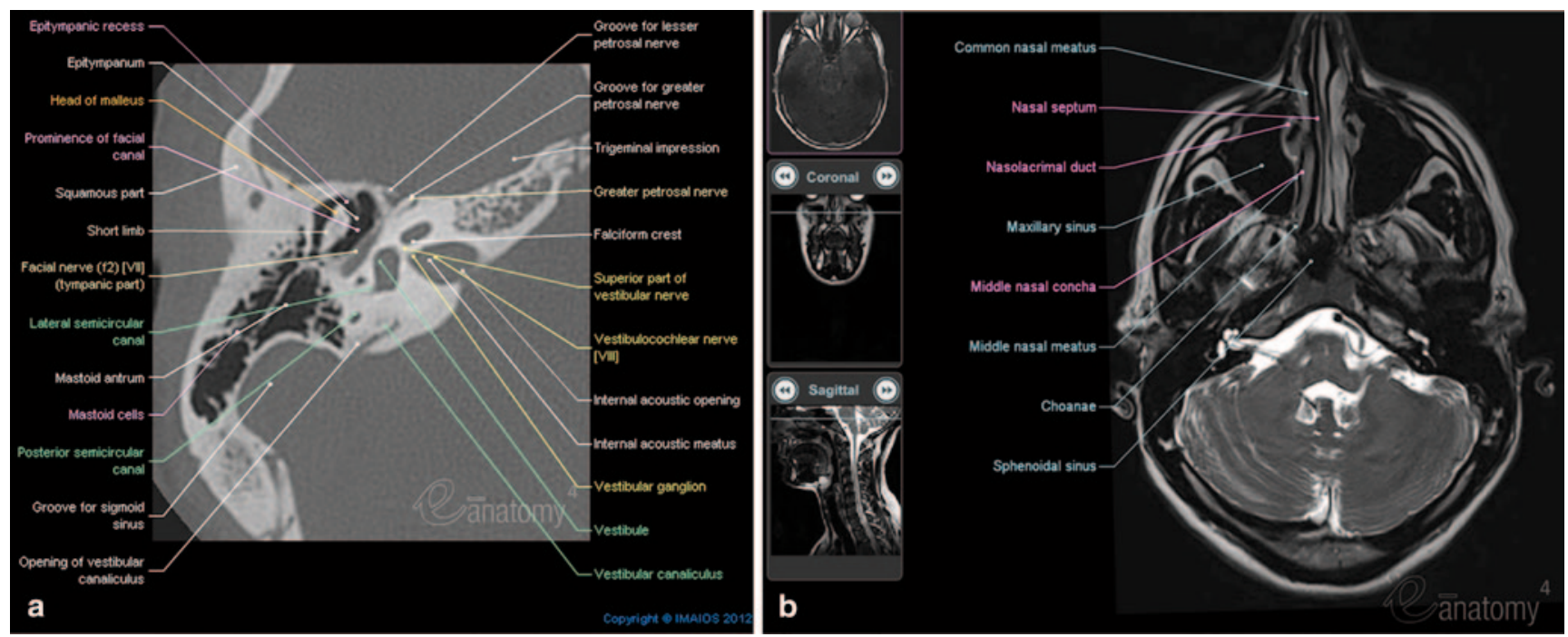

Fig. 2 Examples of the anatomic details that are available (a) CT of the petrous bone (b) MRI of the paranasal sinuses (C) IMAIOS SAS (last time accessed on 6th December 2012)

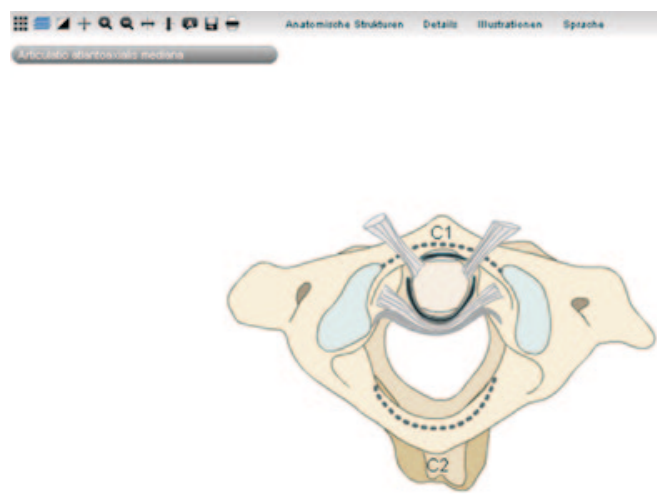

(2) $X=4+Q Q-10 \mathrm{Br}$

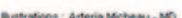

Signal recording

Veroffenticict am Sonntag is fefteus 2009 won Denis Hos

A magnet is a magnetic dipole and it can be represented by a magnetic vector. A moving magnetic field

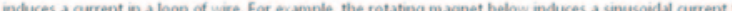
can be recorded.

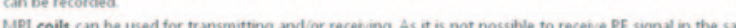
ais as $\mathrm{B} 0$. colls are only sensitive to variations of transverse magnetcation vector. Quadrature $\mathrm{RF}$ colls

(circularty polarced colls) consist of at least two coils that are onented orthogonal to each over (and both are othogonal to BO arisi. They have a better signal to noise ratio than linear PF coils.

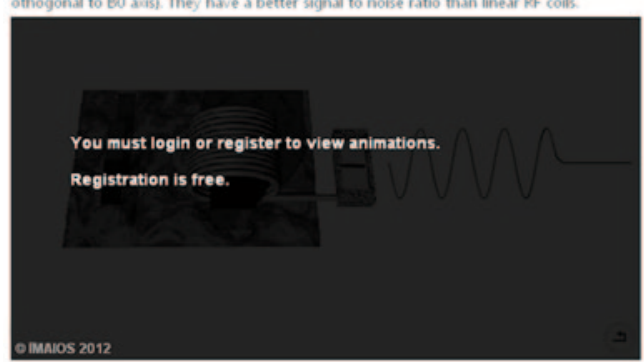

Fig. 3 The advantages of registration. On the left, you see what you get without registration, the pictures on the right show the extended content that is reserved for registered users. Registration is free. Top: The schematic drawings of the spine are freely accessible, but a reg-

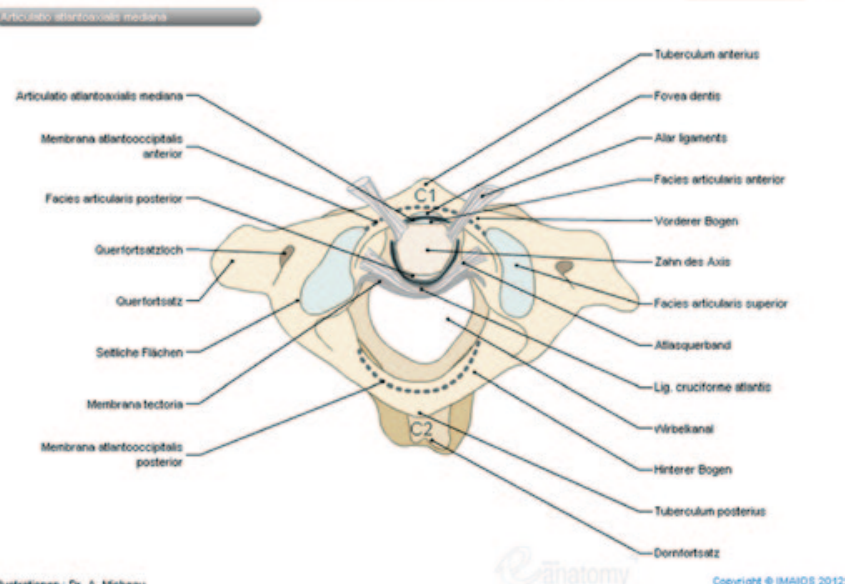

\section{Signal recording}

Veroffentlicht am Sonntag. 15. Februar 2009 von Denis Hoa

A magnet is a magnetic dipole and it can be represented by a magnetic vector. A moving magnetic field induces a current in a loop of wire. For example the rotating magnet below induces a sinusoidal current that can be recorded.

MRI coils can be used for transmitting and/or receiving. As it is not possible to receive RF signal in the same axis as BO. coils are only sensitive to variations of transverse magnetication vector. Quadrature RF coils (circularly polarized coils) consist of at least two coils that are oriented orthogonal to each over (and both are othogonal to $B 0$ axis). They have a better signal to noise ratio than linear $\mathrm{RF}$ colls.

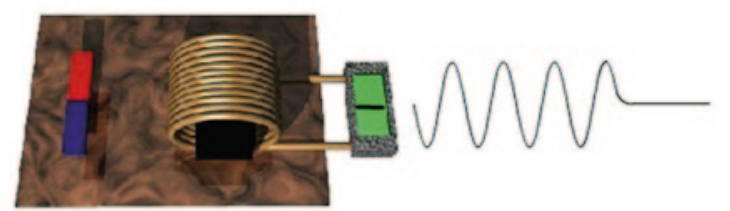

istration is required for the legends. Bottom: The introduction to MRI contains videos that illustrate specific points. The videos are only shown to registered users. (C) IMAIOS SAS (last time accessed on 6th December 2012) 